PROCEEDINGS OF THE

AMERICAN MATHEMATICAL SOCIETY

Volume 137, Number 4, April 2009, Pages 1363-1369

S 0002-9939(08)09614-7

Article electronically published on October 16, 2008

\title{
CONVERGENCE OF WEIGHTED POLYNOMIAL MULTIPLE ERGODIC AVERAGES
}

\author{
QING CHU
}

(Communicated by Bryna Kra)

\begin{abstract}
In this article we study weighted polynomial multiple ergodic averages. A sequence of weights is called universally good if any polynomial multiple ergodic average with this sequence of weights converges in $L^{2}$. We find a necessary condition and show that for any bounded measurable function $\phi$ on an ergodic system, the sequence $\phi\left(T^{n} x\right)$ is universally good for almost every $x$. The linear case was covered by Host and Kra.
\end{abstract}

\section{INTRODUCTION}

In his innovative proof of Szemerédi's Theorem via ergodic theory, Furstenberg introduced certain multiple ergodic averages. There have been many results on these and other nonconventional ergodic averages, including the multiple ergodic theorems of Host and Kra [2, 3], Leibman [5, Ziegler [10. Recently Host and Kra studied weighted ergodic theorems for multiple averages along arithmetic progressions. We give a generalization of this result for polynomial averages, showing:

Theorem 1.1. Let $(Y, \nu, S)$ be an ergodic system and $\phi \in L^{\infty}(\nu)$. Then there exists $Y_{0} \subset Y$ with $\nu\left(Y_{0}\right)=1$ such that, for every $y_{0} \in Y_{0}$, every system $(X, \mu, T)$, every $r \geq 1$, all integer polynomials $p_{1}, \ldots, p_{r}$ and all functions $f_{1}, \ldots, f_{r} \in L^{\infty}(\mu)$, the averages

$$
\frac{1}{N} \sum_{n=0}^{N-1} \phi\left(S^{n} y_{0}\right) T^{p_{1}(n)} f_{1} \cdots T^{p_{r}(n)} f_{r}
$$

converge in $L^{2}(\mu)$.

Throughout this article, by integer polynomial we mean a polynomial all of whose coefficients are integers.

The case of $p_{i}(n)=i n$ was proved by Host and Kra [4].

Remark 1.2. One may wonder why in the theorem $S^{n}$ is not replaced by $S^{p(n)}$ for some integer polynomial $p(n)$. In fact, the latter would be much harder to prove, even in the simplest case that $r=1$ and $p_{1}(n)=n$. This problem is equivalent to showing the convergence of the averages of $\phi\left(S^{p(n)} y_{0}\right) e^{2 \pi i n t}$ for all $t \in \mathbb{T}$ and all $y_{0} \in Y_{0}$, where $Y_{0}$ does not depend on $t$. But this problem reduces to a question

Received by the editors February 21, 2008, and, in revised form, April 14, 2008.

2000 Mathematics Subject Classification. Primary 37A05, 37A30.

Key words and phrases. Weighted ergodic averages, universally good sequences, WienerWintner ergodic theorem, nilsequences. 
of almost everywhere convergence of multiple ergodic averages along polynomials, and this question is out of reach for the moment.

Note that the set $Y_{0}$ does not depend on $X$ or on $f_{i}, i=1, \ldots, r$. We say that for every $y_{0} \in Y_{0}$, the sequence $\phi\left(S^{n} y_{0}\right)$ is universally good for the convergence in the mean of polynomial multiple ergodic averages.

For $r=1$ and $p(n)=n$, the result follows immediately from the classical WienerWintner Ergodic Theorem [9] and a corollary of the Spectral Theorem. We follow a similar strategy, generalizing the proof in 4] along arithmetic progressions to polynomial progressions, but we need to address some deeper technical issues.

We first recall some definitions; see [1] and 4] for the details. Let $G$ be a $k$-step nilpotent Lie group and $\Gamma \subset G$ be a discrete, cocompact subgroup of $G$. The compact manifold $X=G / \Gamma$ is called a $k$-step nilmanifold. The Haar measure $\mu$ of $X$ is the unique probability measure invariant under the left translations $x \mapsto g x$ of $G$ on $X$. Letting $T$ denote left multiplication by a fixed element $\alpha \in G$, we call $(X, \mu, T)$ a $k$-step nilsystem. Let $f: X \rightarrow C$ be a continuous function, $x_{0} \in X$; then the sequence $\left(f\left(\alpha^{n} x_{0}\right): n \in \mathbb{Z}\right)$ is called a basic $k$-step nilsequence. The family of basic $k$-step nilsequences forms a subalgebra of $l^{\infty}$. Under the uniform norm $\|\cdot\|_{\infty}$ of $l^{\infty}$, we call a uniform limit of basic $k$-step nilsequences a $k$-step nilsequence.

The proof of Theorem 1.1 is broken down into two pieces. First we give a convergence criterion for weighted polynomial multiple ergodic averages.

Theorem 1.3 (Convergence Criterion for Weighted Averages). For any $r, b \in \mathbb{N}$, there exists an integer $K \geq 1$ with the following property: for any bounded sequence $\mathbf{c}=\left(c_{n}: n \in \mathbb{Z}\right)$, if the averages

$$
\frac{1}{N} \sum_{n=0}^{N-1} c_{n} d_{n}
$$

converge as $N \rightarrow \infty$ for every $K$-step nilsequence $\boldsymbol{d}=\left(d_{n}: n \in \mathbb{Z}\right)$, then for every system $(X, \mu, T)$, all $f_{1}, \ldots, f_{r} \in L^{\infty}(X)$, and all integer polynomials $p_{1}, \ldots, p_{r}$ of degree $\leq b$, the averages

$$
\frac{1}{N} \sum_{n=0}^{N-1} c_{n} T^{p_{1}(n)} f_{1} \cdot T^{p_{2}(n)} f_{2} \cdots T^{p_{r}(n)} f_{r}
$$

converge in $L^{2}(X)$.

The bulk of this paper is devoted to the proof of this theorem. Then our main result follows from the following Generalized Wiener-Wintner Theorem proved by Host and Kra in 44. The case of a polynomial version of the Wiener-Wintner theorem was proved by Lesigne ([7], [8]).

Theorem 1.4 (Generalized Wiener-Wintner Theorem [4). Let $(X, \mu, T)$ be an ergodic system and $\phi$ be a bounded measurable function on $X$. Then there exists $X_{0} \subset X$ with $\mu\left(X_{0}\right)=1$ such that for every $x \in X_{0}$, the averages

$$
\frac{1}{N} \sum_{n=0}^{N-1} \phi\left(T^{n} x\right) b_{n}
$$

converge as $N \rightarrow \infty$ for every $x \in X_{0}$ and every nilsequence $b=\left(b_{n}: n \in \mathbb{Z}\right)$. 
While nilsequences do not appear in the statement of Theorem 1.1, they are used as tools in its proof. Both Theorems 1.3 and 1.4 are of interest on their own, as results on nilsequences.

\section{Proof of Theorem 1.3}

Using a standard ergodic decomposition argument, it suffices to prove Theorem 1.3 for ergodic systems.

2.1. We first remind the reader of a definition from Leibman's paper 6]. We call a sequence $\{g(n)\}_{n \in \mathbb{Z}}$ with values in a nilpotent group $G$ a polynomial sequence if $g(n)$ is of the form $g(n)=a_{1}^{p_{1}(n)} \ldots a_{m}^{p_{m}(n)}$, where $a_{1}, \ldots, a_{m} \in G$ and $p_{1}, \ldots, p_{m}$ are polynomials taking integer values on the integers.

Before stating the next proposition, we explain briefly its meaning: we can view the sequence of values of a continuous function along a polynomial sequence on a nilmanifold as the sequence of values of some other continuous function along an ordinary orbit of some other nilsystem.

Proposition 2.1. Let $(X=G / \Gamma, T)$ be a nilsystem, $x_{0} \in X, p$ be an integer polynomial, and $f \in \mathcal{C}(X)$. Then there exists a nilsystem $(Y, S), y_{0} \in Y, h \in \mathcal{C}(Y)$, such that $f\left(T^{p(n)} x_{0}\right)=h\left(S^{n} y_{0}\right)$ for every $n$.

Proof. Let $(X=G / \Gamma, T)$ be a nilsystem. Suppose $T x:=\alpha x$, for some $\alpha \in G$. Then $T^{p(n)} x=\alpha^{p(n)} x$. Let $g(n):=\alpha^{p(n)}$; then $g$ is a polynomial sequence in $G$. Let $\pi: G \rightarrow X$ be the factorization mapping. We will assume that $x_{0}=\pi\left(\mathbf{1}_{G}\right)$; otherwise if $x_{0}=\pi(\gamma), \gamma \in G$, we write $g(n) x_{0}=g(n) \gamma \pi\left(\mathbf{1}_{G}\right)$ and replace $g(n)$ by $g(n) \gamma$.

Now we have a nilpotent Lie group $G$, a discrete cocompact subgroup $\Gamma$ and a polynomial sequence $g$ in $G$. By Proposition 3.14 in Leibman's paper [6, there exist a nilpotent Lie group $\widetilde{G}$, a discrete cocompact subgroup $\widetilde{\Gamma}$, an epimorphism $\eta: \widetilde{G} \rightarrow G$ with $\eta(\widetilde{\Gamma}) \subseteq \Gamma$, a unipotent automorphism $\widetilde{\tau}$ of $\widetilde{G}$ with $\widetilde{\tau}(\widetilde{\Gamma})=\widetilde{\Gamma}$, and an element $\tilde{c} \in \widetilde{G}$ such that

$$
g(n)=\eta\left(\widetilde{\tau}^{n}(\tilde{c})\right), \quad n \in \mathbb{Z} .
$$

Let $\widetilde{X}=\widetilde{G} / \widetilde{\Gamma}$ and let $\widetilde{\pi}: \widetilde{G} \rightarrow \widetilde{X}$ be the factorization mapping.

The epimorphism $\eta: \widetilde{G} \rightarrow G$ factors to a map $\widetilde{X} \rightarrow X$ (we also denote it by $\eta$ ), which is onto and satisfies

$$
\pi \circ \eta=\eta \circ \tilde{\pi}
$$

The map $\widetilde{\tau}$ induces a homomorphism $\widetilde{X} \rightarrow \widetilde{X}$, which we also denote by $\widetilde{\tau}$. It satisfies

Let $\widetilde{x}_{0}=\widetilde{\pi}\left(\mathbf{1}_{\widetilde{G}}\right) ;$ then

$$
\widetilde{\tau} \circ \tilde{\pi}=\tilde{\pi} \circ \widetilde{\tau}
$$

$$
\eta\left(\widetilde{\tau}^{n}\left(\tilde{c} \widetilde{x}_{0}\right)\right)=g(n) x_{0}, n \in \mathbb{Z} .
$$

Let $\widehat{G}$ be the extension of $\widetilde{G}$ by $\widetilde{\tau}$. Then $\widehat{G}$ is a nilpotent Lie group (see Proposition 3.9 in [6] ). Let $\widehat{\tau}$ be the element in $\widehat{G}$ representing $\widetilde{\tau}$, so that $\widetilde{\tau}(\widetilde{\alpha})=\widehat{\tau} \widetilde{\alpha} \widehat{\tau}^{-1}$ for any $\widetilde{\alpha} \in \widetilde{G}$, where the multiplication of $\widehat{G}$ is given by this formula. We have $\widehat{G}=\left\{\widetilde{g} \widehat{\tau}^{n}: \widetilde{g} \in \widetilde{G}, n \in \mathbb{Z}\right\}$ and $\widetilde{G}$ is open in $\widehat{G}$.

Let $\widehat{\Gamma}$ be the subgroup of $\widehat{G}$ spanned by $\widetilde{\Gamma}$ and $\widehat{\tau}$. As $\widetilde{\tau}(\widetilde{\Gamma})=\widetilde{\Gamma}$, we have $\widehat{\Gamma}=$ $\left\{\widetilde{\gamma} \widehat{\tau}^{n}: \widetilde{\gamma} \in \widetilde{\Gamma}, n \in \mathbb{Z}\right\}$ and $\widehat{\Gamma} \cap \widetilde{G}=\widetilde{\Gamma}$. By the definition of the relative topology, 
$\widehat{\Gamma}$ is a discrete subgroup of $\widehat{G}$. Moreover, $\widehat{G} / \widehat{\Gamma}=(\widetilde{G} \widehat{\Gamma} / \widehat{\Gamma})$ can be identified with $\widetilde{G} /(\widehat{\Gamma} \cap \widetilde{G})=\widetilde{G} / \widetilde{\Gamma}=\widetilde{X}$. We write $\widehat{\pi}: \widehat{G} \rightarrow \widetilde{X}$ for the quotient map.

Let $\widetilde{x} \in \widetilde{X}$ and $\tilde{g} \in \widetilde{G}$ with $\widetilde{\pi}(\tilde{g})=\widetilde{x}$. We have

$$
\widetilde{\tau}(\widetilde{x})=\widetilde{\pi}(\widetilde{\tau}(\tilde{g}))=\widehat{\pi}\left(\widehat{\tau} \tilde{g} \widetilde{\tau}^{-1}\right)=\widehat{\pi}(\widehat{\tau} \tilde{g})=\widehat{\tau} \widetilde{x},
$$

because $\widehat{\tau}^{-1} \in \widehat{\Gamma}$. So for every $n$,

$$
g(n) x_{0}=\eta\left(\widetilde{\tau}^{n}\left(\tilde{c} \widetilde{x}_{0}\right)\right)=\eta\left(\widehat{\tau^{n}}\left(\tilde{c} \widetilde{x}_{0}\right)\right) .
$$

Let $Y=(\widehat{G} / \widehat{\Gamma}, S)=(\widetilde{X}, S), S \widetilde{x}=\widehat{\tau} \widetilde{x}$, and let $h=f \circ \eta$, and $y_{0}=\tilde{c} \widetilde{x}_{0}$. This system and this function satisfy the announced properties.

2.2. We recall a few properties of the seminorms and the factors introduced in 2 . Let $(X, \mu, T)$ be an ergodic system. For an integer $k \geq 0$, we write $X^{[k]}=X^{2^{k}}$ and $T^{[k]}: X^{[k]} \rightarrow X^{[k]}$ for the map $T \times T \times \ldots \times T$, taken $2^{k}$ times. We define by induction a probability measure $\mu^{[k]}$ on $X^{[k]}$ that is invariant under $T^{[k]}$. Set $\mu^{[0]}=\mu$. For $k \geq 0$, let $\mathcal{I}^{[k]}$ be the $\sigma$-algebra of $T^{[k]}$-invariant subsets of $X^{[k]}$. Then $\mu^{[k+1]}$ is the relatively independent product of $\mu^{[k]}$ over $\mathcal{I}^{k}$, which means for $F, F^{\prime} \in L^{\infty}\left(X^{[k]}\right)$,

$$
\int_{X^{[k+1]}} F \otimes F^{\prime} d \mu^{[k+1]}=\int_{X^{[k]}} \mathbb{E}\left(F \mid \mathcal{I}^{[k]}\right) \mathbb{E}\left(F^{\prime} \mid \mathcal{I}^{[k]}\right) d \mu^{[k]} .
$$

For a bounded measurable function $f$, we define

$$
\|f\|_{k}=\left(\int_{X^{[k]}} \prod_{\varepsilon \in\{0,1\}^{k}} C^{|\varepsilon|} f\left(x_{\varepsilon}\right) d \mu^{[k]}(x)\right)^{1 / 2^{k}},
$$

where $C: \mathbb{C} \rightarrow \mathbb{C}$ is the conjugacy map $z \mapsto \bar{z}, \varepsilon=\varepsilon_{1} \varepsilon_{2} \ldots \varepsilon_{k}$ with $\varepsilon_{i} \in\{0,1\}$ and $|\varepsilon|=\varepsilon_{1}+\varepsilon_{2}+\cdots+\varepsilon_{k}$. It is shown in [2] that for every $k \geq 1,\|\cdot\|_{k}$ is a seminorm on $L^{\infty}(\mu)$.

Moreover, for every $k \geq 2, X$ admits a factor $Z_{k-1}$ such that, for every $f \in$ $L^{\infty}(\mu),\|f\|_{k}=0$ if and only if $\mathbb{E}\left(f \mid Z_{k-1}\right)=0$. One of the main results of [2] is that, for every $k, Z_{k}$ is an inverse limit of $k$-step nilsystems. We call this result the Structure Theorem.

Let $\left(Z=Z_{1}(X), m, T\right)$ be the Kronecker factor of $(X, \mu, T)$. For $s \in Z$, we define a measure $\mu_{s}$ on $X \times X$ by

$$
\int_{X \times X} f(x) f^{\prime}\left(x^{\prime}\right) d \mu_{s}\left(x, x^{\prime}\right)=\int_{Z} \mathbb{E}(f \mid Z)(z) \cdot \mathbb{E}\left(f^{\prime} \mid Z\right)(s z) d m(z) .
$$

For every $s \in Z$ the measure $\mu_{s}$ is invariant under $T \times T$ and is ergodic for $m$-almost every $s$. The ergodic decomposition of $\mu \times \mu$ under $T \times T$ is

$$
\mu \times \mu=\int_{Z} \mu_{s} d m(s) .
$$

For each $s \in Z$ such that $\left(X \times X, \mu_{s}, T \times T\right)$ is ergodic, and for each integer $k \geq 1$, a measure $\left(\mu_{s}\right)^{[k]}$ on $(X \times X)^{[k]}$ can be defined in the same way as $\mu^{[k]}$. Furthermore, a seminorm $\|\cdot\|_{k, s}$ on $L^{\infty}\left(\mu_{s}\right)$ can be associated to this measure in the same way as the seminorm $\|\cdot\|_{k}$ is associated to $\mu^{[k]}$. It follows from the definition (2) that for every $f \in L^{\infty}(\mu)$,

$$
\|f\|_{k+1}^{2^{k+1}}=\int_{Z}\|f \otimes \bar{f}\|_{k, s}^{2^{k}} d m(s) .
$$


2.3. We return to the proof of Theorem 1.3. We may assume that the polynomials $p_{1}, \ldots, p_{r}$ are nonconstant and essentially distinct; that is, $p_{i}-p_{j} \neq$ constant for $i \neq j$.

The following theorem will be proved in the next section.

Theorem 2.2. For any $r, b \in \mathbb{N}$, there exists $k \in \mathbb{N}$, such that for any nonconstant essentially distinct polynomials $p_{1}, \ldots, p_{r}: \mathbb{Z} \rightarrow \mathbb{Z}$ of degree $\leq b$, for every ergodic system $(X, \mu, T)$, every $f_{1}, \ldots, f_{r} \in L^{\infty}(X)$ with $\left\|f_{1}\right\|_{k}=0$, and any bounded sequence $\mathbf{c}=\left(c_{n}: n \in \mathbb{Z}\right)$, one has

$$
\lim _{N \rightarrow \infty}\left\|\frac{1}{N} \sum_{n=0}^{N-1} c_{n} T^{p_{1}(n)} f_{1} \cdots T^{p_{r}(n)} f_{r}\right\|_{L^{2}(X)}=0 .
$$

2.4. Now we give the proof of Theorem 1.3 ,

Proof of Theorem 1.3 from Proposition 2.1 and Theorem 2.2. The proof is exactly the same as the proof of Theorem 2.24 in 4 . For any $r, b \in \mathbb{N}$, let $k \in \mathbb{N}$ be the integer in Theorem 2.2. and let $Z_{k-1}$ be the $(k-1)$-th factor of $(X, \mu, T)$, as given by the Structure Theorem. By definition, if $\mathbb{E}\left(f_{1} \mid Z_{k-1}\right)=0$, then $\left\|f_{1}\right\|_{k}=0$, and by Theorem 2.2, the averages (44) converge to zero in $L^{2}(X)$. We say that the factor $Z_{k-1}$ is the characteristic for the convergence of these averages. Therefore, it suffices to prove the result when the functions are measurable with respect to the factor $Z_{k-1}$.

Since $Z_{k-1}$ is an inverse limit of $(k-1)$-step nilsystems, by density, we can assume that $(X, \mu, T)$ is a $(k-1)$-step nilsystem and that the functions $f_{1}, \ldots, f_{r}$ are continuous.

But in this case, by Proposition 2.1, for every $x \in X$, and all polynomials $p_{1}, \ldots, p_{r}$, there exist nilsystems $\left(Y_{1}, S_{1}\right), \ldots,\left(Y_{r}, S_{r}\right), y_{i} \in Y_{i}$, and $g_{i} \in \mathcal{C}\left(Y_{i}\right)$, such that $f_{i}\left(T^{p_{i}(n)} x\right)=g_{i}\left(S_{i}^{n} y_{i}\right), i=1, \ldots, r$.

Let $K$ be the maximal order of the nilsystems $\left(Y_{i}, S_{i}\right), i=1, \ldots, r$. Then the system $\left(Y=Y_{1} \times \cdots \times Y_{r}, S=S_{1} \times \cdots \times S_{r}\right)$ is a $K$-step nilsystem. Let $g$ : $Y_{1} \times \cdots \times Y_{r} \rightarrow \mathbb{R}$ be given by $g(y)=g\left(y_{1}, \ldots, y_{r}\right)=g_{1}\left(y_{1}\right) \cdot \ldots \cdot g_{r}\left(y_{r}\right)$. So the sequence

$$
\begin{aligned}
& \left\{f_{1}\left(T^{p_{1}(n)} x\right) \cdot f_{2}\left(T^{p_{2}(n)} x\right) \cdot \ldots \cdot f_{r}\left(T^{p_{r}(n)} x\right)\right\}_{n \in \mathbb{Z}} \\
= & \left\{g_{1}\left(S_{1}^{n} y_{1}\right) \cdot g_{2}\left(S_{2}^{n} y_{2}\right) \cdot \ldots \cdot g_{r}\left(S_{r}^{n} y_{r}\right)\right\}_{n \in \mathbb{Z}} \\
= & \left\{g\left(S^{n} y\right)\right\}_{n \in \mathbb{Z}}
\end{aligned}
$$

is a $K$-step nilsequence and by hypothesis, the averages (11) converge for every $x \in X$.

\section{Proof of Theorem 2.2}

Note that our goal is very similar to the main result in Leibman's paper [5], the only difference being that in our case we are dealing with the weighted averages. In fact, we can deduce Theorem 2.2 by following very closely the arguments of Leibman in his paper to cover our weighted case with some modifications. But here we adopt another way that allows us to deduce it directly from Leibman's result.

Proof of Theorem 2.2. We prove the result by several steps.

(i) The following assertion follows immediately from Theorem 3 in Leibman's paper [5]: 
Let $r, b \in \mathbb{N}$ be fixed. There exists an integer $k=k(r, b)$ such that for every family of nonconstant essentially distinct polynomials $p_{1}, \ldots, p_{r}: \mathbb{Z}^{2} \rightarrow \mathbb{Z}$ of degree $\leq b$, we have: for every ergodic system $(X, \mathcal{B}, \mu, T)$, and every $f_{1}, \ldots, f_{r} \in L^{\infty}(X)$ with $\left\|f_{1}\right\|_{k}=0$, one has

$$
\lim _{N \rightarrow \infty}\left\|\frac{1}{N^{2}} \sum_{0 \leq m, n<N} T^{p_{1}(m, n)} f_{1} \cdots T^{p_{r}(m, n)} f_{r}\right\|_{L^{2}(X)}=0 .
$$

(ii) We use the notation of Section 2.2. It follows from (3) that $\left\|f_{1}\right\|_{k+1}=0$ implies $\left\|f_{1} \otimes \bar{f}_{1}\right\|_{k, s}=0$ for $m$-almost every $s$. Using the previous result to the ergodic system $\left(X \times X, \mu_{s}, T \times T\right)$, one gets: if $\left\|f_{1}\right\|_{k+1}=0$, then

$$
\begin{aligned}
& \lim _{N \rightarrow \infty} \frac{1}{N^{2}} \sum_{0 \leq m, n<N}\left|\int T^{p_{1}(m, n)} f_{1} \cdots T^{p_{r}(m, n)} f_{r} d \mu\right|^{2} \\
= & \lim _{N \rightarrow \infty} \frac{1}{N^{2}} \sum_{0 \leq m, n<N}\left|\int_{Z} \int_{X \times X}(T \times T)^{p_{1}(m, n)} f_{1} \otimes \bar{f}_{1} \cdots(T \times T)^{p_{r}(m, n)} f_{r} \otimes \bar{f}_{r} d \mu_{s} d m(s)\right| \\
\leq & \int_{Z} \lim _{N \rightarrow \infty} \int_{X \times X}\left|\frac{1}{N^{2}} \sum_{0 \leq m, n<N}(T \times T)^{p_{1}(m, n)} f_{1} \otimes \bar{f}_{1} \cdots(T \times T)^{p_{r}(m, n)} f_{r} \otimes \bar{f}_{r}\right| d \mu_{s} d m(s) \\
\leq & \int_{Z} \lim _{N \rightarrow \infty}\left\|\frac{1}{N^{2}} \sum_{0 \leq m, n<N}(T \times T)^{p_{1}(m, n)} f_{1} \otimes \bar{f}_{1} \cdots(T \times T)^{p_{r}(m, n)} f_{r} \otimes \bar{f}_{r}\right\|_{L^{2}\left(\mu_{s}\right)} d m(s)=0 .
\end{aligned}
$$

(iii) Expanding the square, one sees that

$$
\left\|\frac{1}{N} \sum_{n=0}^{N-1} c_{n} T^{p_{1}(n)} f_{1} \cdots T^{p_{r}(n)} f_{r}\right\|_{L^{2}}^{2}
$$

is equal to

$$
\frac{1}{N^{2}} \sum_{0 \leq m, n<N} c_{n} \bar{c}_{m} \int T^{p_{1}(n)} f_{1} \cdots T^{p_{r}(n)} f_{r} T^{p_{1}(m)} \bar{f}_{1} \cdots T^{p_{r}(m)} \bar{f}_{r} d \mu,
$$

which is less than a constant times

$$
\frac{1}{N^{2}} \sum_{0 \leq m, n<N}\left|\int T^{p_{1}(n)} f_{1} \cdots T^{p_{r}(n)} f_{r} T^{p_{1}(m)} \bar{f}_{1} \cdots T^{p_{r}(m)} \bar{f}_{r} d \mu\right|,
$$

since by our assumption the sequence $c_{n}$ is bounded. Notice that $p_{1}(n), \ldots$, $p_{r}(n), p_{1}(m), \ldots, p_{r}(m)$ is a family of $2 r$ essentially distinct polynomials of degree at most $b$.

(iv) Combining the previous parts we see that if $\left\|f_{1}\right\|_{k(2 r, b)+1}=0$, where $k(r, b)$ is defined in part (i), then for every family of nonconstant essentially distinct polynomials $p_{1}, \ldots, p_{r}: \mathbb{Z} \rightarrow \mathbb{Z}$ of degree $\leq b$, we have that the average (5) converges to 0 . This proves Theorem 2.2 .

\section{ACKNowledgement}

We thank the referee for very useful remarks and the simplification of the proof. 


\section{REFERENCES}

1. V. Bergelson, B. Host and B. Kra, with an appendix by I. Ruzsa. Multiple recurrence and nilsequences. Inventiones Math. 160 (2005), 261-303. MR2138068 (2007i:37009)

2. B. Host and B. Kra. Nonconventional ergodic averages and nilmanifolds. Ann. Math. 161 (2005), 397-488. MR2150389 (2007b:37004)

3. B. Host and B. Kra. Convergence of polynomial ergodic averages. Israel J. Math. 149 (2005), 1-19. MR2191208 (2007c:37004)

4. B. Host and B. Kra. Uniformity seminorms on $\ell^{\infty}$ and applications. Available at http://arxiv.org/abs/0711.3637v1.

5. A. Leibman. Convergence of multiple ergodic averages along polynomials of several variables. Israel J. Math. 146 (2005), 303-315. MR2151605 (2006c:28016)

6. A. Leibman. Pointwise convergence of ergodic averages for polynomial sequences of translations on a nilmanifold. Ergodic Th. and Dyn. Sys. 25 (2005), 201-213. MR2122919 (2006j:37004)

7. E. Lesigne. Un théorème de disjonction de systèmes dynamiques et une généralisation du théorème ergodique de Wiener-Wintner. Ergodic Th. and Dyn. Sys. 10 (1990), 513-521. MR.1074316 (91h:28016)

8. E. Lesigne. Spectre quasi-discret et théorème ergodique de Wiener-Wintner pour les polynômes. Ergodic. Th. and Dyn. Sys. 13 (1993), 767-784. MR.1257033 (95e:28012)

9. N. Wiener and A. Wintner. Harmonic analysis and ergodic theory. Amer. J. Math. 63 (1941), 415-426. MR0004098 (2:319b)

10. T. Ziegler. A non-conventional ergodic theorem for a nilsystem. Ergodic Th. and Dyn. Sys. 25 (2005), 1357-1370. MR.2158410 (2006d:37009)

Université Paris-Est, Laboratoire d'Analyse et de Mathématiques Appliquées, UMR CNRS 8050, 5 Bd Descartes, 77454 Marne la Vallée Cedex 2, France

E-mail address: qing.chu@univ-mlv.fr 